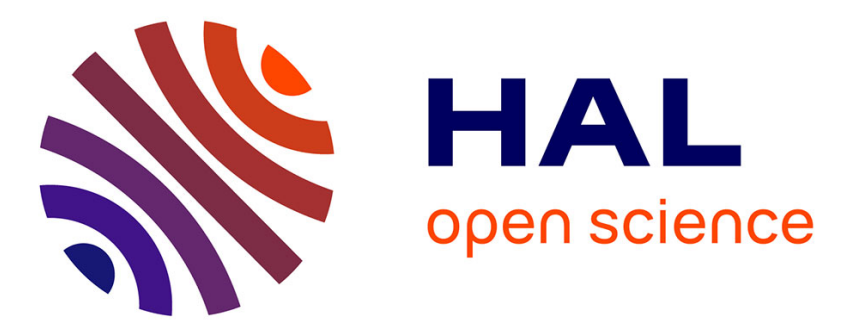

\title{
Motion sickness evaluation and comparison for a static driving simulator and a dynamic driving simulator
}

Baris Aykent, Frédéric Merienne, Christophe Guillet, Damien Paillot, Andras Kemeny

\section{- To cite this version:}

Baris Aykent, Frédéric Merienne, Christophe Guillet, Damien Paillot, Andras Kemeny. Motion sickness evaluation and comparison for a static driving simulator and a dynamic driving simulator. Proc. IMechE, Part D: Journal of Automobile Engineering, 2014, pp.1-12. 10.1177/0954407013516101 . hal-00981166v2

\author{
HAL Id: hal-00981166 \\ https://hal.science/hal-00981166v2
}

Submitted on 28 Apr 2014

HAL is a multi-disciplinary open access archive for the deposit and dissemination of scientific research documents, whether they are published or not. The documents may come from teaching and research institutions in France or abroad, or from public or private research centers.
L'archive ouverte pluridisciplinaire HAL, est destinée au dépôt et à la diffusion de documents scientifiques de niveau recherche, publiés ou non, émanant des établissements d'enseignement et de recherche français ou étrangers, des laboratoires publics ou privés. 


\title{
Motion sickness evaluation and comparison for a static driving simulator and a dynamic driving simulator
}

\author{
Baris Aykent', Frederic Merienne', Christophe Guillet', \\ Damien Paillot' and Andras Kemeny ${ }^{1,2}$
}

\begin{abstract}
This paper deals with driving simulation and in particular with the important issue of motion sickness. The paper proposes a methodology to evaluate the objective illness rating metrics deduced from the motion sickness dose value and questionnaires for both a static simulator and a dynamic simulator. Accelerations of the vestibular cues (head movements) of the subjects were recorded with and without motion platform activation. In order to compare user experiences in both cases, the head-dynamics-related illness ratings were computed from the obtained accelerations and the motion sickness dose values. For the subjective analysis, the principal component analysis method was used to determine the conflict between the subjective assessment in the static condition and that in the dynamic condition. The principal component analysis method used for the subjective evaluation showed a consistent difference between the answers given in the sickness questionnaire for the static platform case from those for the dynamic platform case. The two-tailed Mann-Whitney $U$ test shows the significance in the differences between the self-reports to the individual questions. According to the two-tailed Mann-Whitney $U$ test, experiencing nausea $(p=0.019<0.05)$ and dizziness $(p=$ $0.018<0.05)$ decreased significantly from the static case to the dynamic case. Also, eye strain $(p=0.047<0.05)$ and tiredness $(p=0.047<0.05)$ were reduced significantly from the static case to the dynamic case. For the perception fidelity analysis, the Pearson correlation with a confidence interval of $95 \%$ was used to study the correlations of each question with the $x$ illness rating component $\mathbb{R}_{x}$, the $y$ illness rating component $\mathbb{R}_{y}$ the $z$ illness rating component $\mathbb{R}_{z}$ and the compound illness rating $\mathbb{I}_{\text {tot }}$. The results showed that the longitudinal head dynamics were the main element that induced discomfort for the static platform, whereas vertical head movements were the main factor to provoke discomfort for the dynamic platform case. Also, for the dynamic platform, lateral vestibular-level dynamics were the major element which caused a feeling of fear.
\end{abstract}

\section{Keywords}

Driving simulator, driver's perception, inertial cue, vestibular-level dynamics, motion sickness

\section{Introduction}

Sensorial cues (visual, auditory, haptic, inertial, vestibular and neuromuscular) play important roles in representing a proper perception in driving simulators. ${ }^{1} \mathrm{~A}$ driving simulator aims to give the sensation of driving as in a real case. To fulfil this objective, the driving simulator must enhance the virtual immersion of the subject in a driving situation. For that reason, restituting the inertial cues on driving simulators is important in order to achieve certain goals by simulations, recognizing that not all simulators can achieve this. ${ }^{2}$ Because of the restricted workspace, it is not possible to represent the vehicle dynamics continuously with a oneto-one scale on the motion platform. Nevertheless, the most desired aim is to minimize the deviation between

\footnotetext{
'Le Laboratoire d'Electronique, d'Informatique et de I'Image (Unité Mixte de Recherche 6306 du CNRS), Arts et Métiers Paris Tech, Chalon-surSaône, France

${ }^{2}$ Technical Centre for Simulation, Renault, Guyancourt, France
}

\section{Corresponding author:}

Baris Aykent, Le Laboratoire d'Electronique, d'Informatique et de l'Image (Unité Mixte de Recherche 6306 du CNRS), Arts et Métiers Paris Tech, 2 Rue T Dumorey, 7I 100 Chalon-sur-Saône, France.

Email: b.aykent@gmail.com 


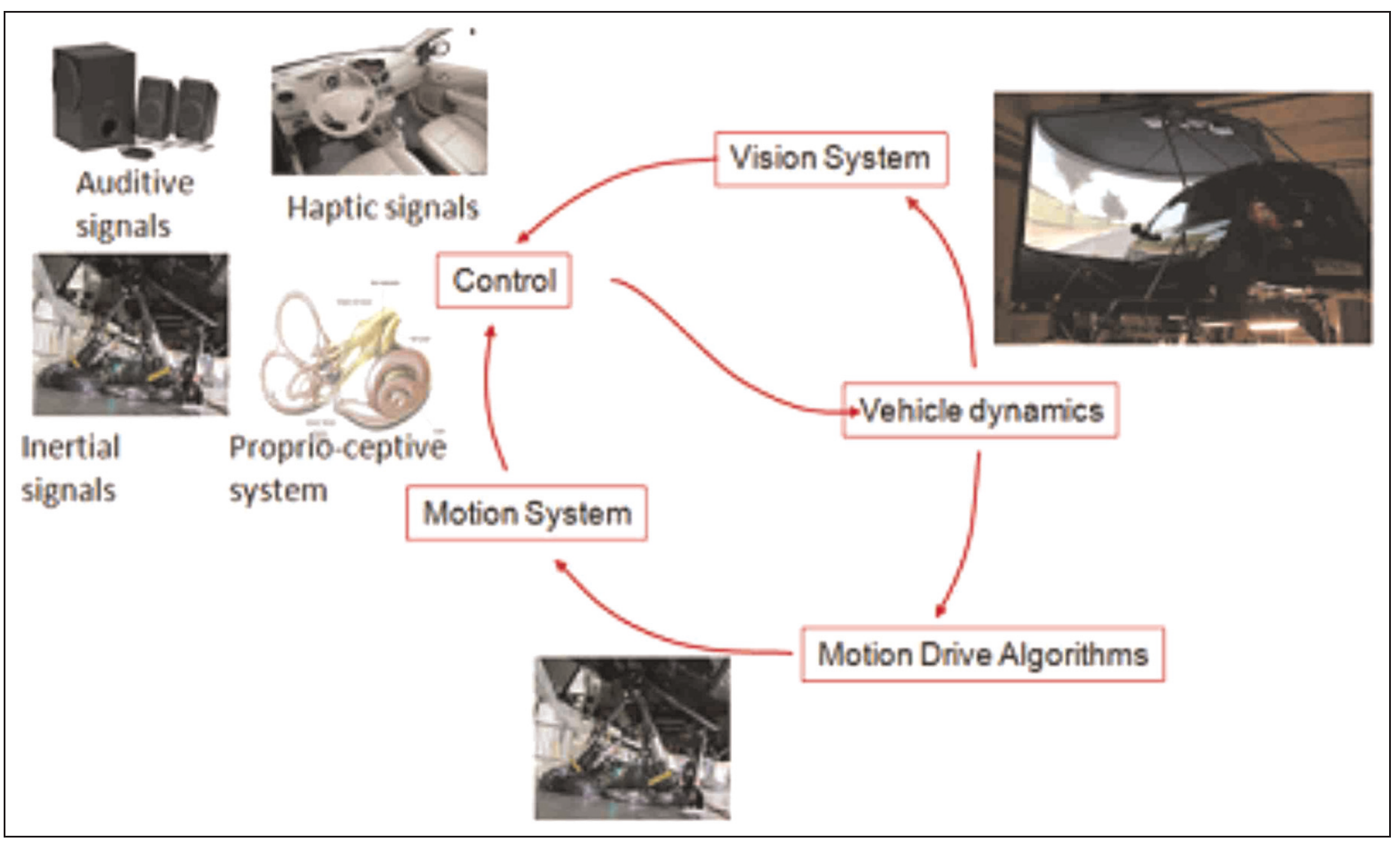

Figure I. Structure of the closed-loop control of the dynamic driving simulator. ${ }^{8}$

the accelerations from the visually (vehicle model) represented dynamics and those from the inertially (motion platform) represented dynamics as realistically as possible depending on the driving task. ${ }^{3}$

Driving simulation sickness has been assessed using dynamic and static simulators in some studies. ${ }^{4-6}$ For a braking manoeuvre, Siegler et al. ${ }^{7}$ stated that, if the motion platform is activated, the bias in reaching increased levels of decelerations is reduced in comparison with the case when the motion platform is inactivated. This indicates a more realistic representation of the vehicle dynamics, which can be interpreted as a reducing effect on motion sickness. In order to reduce the simulator sickness, the difference between the accelerations through the visual and the vestibular cues has to be minimized..$^{3,6,7}$ The vestibular cues correspond to the head movements of the subjects during the driving simulator experiments. The dynamics of the vestibular cues are defined by the longitudinal, lateral, vertical and vectorial compound dynamics (see Figure 1 for the multi-sensory integration and motion sickness mechanisms). Here, the illness ratings are defined as follows: the component illness ratings refer to the illness ratings induced by the longitudinal dynamics, the lateral dynamics and the vertical dynamics separately, whereas the compound illness rating corresponds to the vectorial compound of the longitudinal, lateral and vertical illness ratings. The aim of this paper is to address simulator motion sickness as a correlated function of the component illness ratings and the compound illness ratings for vestibular cues (head movements) using sickness questionnaires. This research work was performed under static and dynamic operations using the driving simulator called the Simulateur Automobile Arts et Metiers (SAAM).

The objectives of this work are to propose a bimodal measurement approach to obtain objective and subjective data, and their correlation with each other to monitor the sickness level of the test subjects during the driving session, first, as a method to determine the motion sickness components (longitudinal, lateral, vertical or total) which are beneficial to representing the motion sickness of the test subjects as an objective measure (namely the illness rating), second, as a technique to measure subjective impressions of the test participants through a motion sickness questionnaire and, third, for correlation between the subjective and the objective data.

This paper aims to investigate the significance level of the following hypotheses. Is there any significant correlation between the vestibular-level illness rating (objective measure) and the perception of sickness (subjective measure) in the case of the static simulator and in the case of the dynamic simulator? Is there any perceptual difference between the static simulator and the dynamic simulator in terms of motion sickness? (Here, the perceptual difference refers to the subjective evaluation difference for the static operation and for the dynamic operation of the simulator motion platform regarding our simulator sickness questionnaire (see Tables 3 and 4 later).)

Potential factors inducing the sickness in virtual reality systems can be split into three major groups: ${ }^{9}$ individual factors, simulator factors and simulated task factors.

Individual factors refer to the sensitivity to the simulator conditions (adaptation), the postural stability, etc. 
In general, they address the driver and his or her behaviour during the driving session (Figure 1). Simulator factors refer to whether the simulator has a motion platform or not (a dynamic driving simulator or static driving simulator, seen as the driving simulator technologies in Figure 1). Simulated task factors refer to the head movements (the vestibular reaction of the driver) with respect to the road scenario, the vehicle dynamics and the driving simulator technologies used for the experimental trials. Two types of driving simulator commonly used are dynamic simulators (moving-base simulators) and static simulators (fixed-base simulators). It is reported that simulator sickness is less likely to occur in moving-base driving simulators. ${ }^{4,10-14}$ Previously, when dynamic simulators and static simulators have been compared, it has been suggested that there is a relation between the illness and the head movements of the pilot. ${ }^{14}$ The motion sickness mechanisms in a driving situation can be summarized by Figure 1. According to Figure 1, the road scenario and the vehicle dynamics model are formed in the visual system of the driving simulator. Compared with the static platform case, the dynamic platform condition also has a hexapod motion platform and motion cueing algorithms (see Figure 1) which convert the vehicle dynamics to restricted platform dynamics. In the study that we are currently presenting here, the contribution of the motion platform to motion sickness is investigated. Apart from the dynamic systems, other cues (auditory cues for engine and traffic sounds, vision system restitution to feed back the driven environment, and force feedback steering wheel as haptic cues to provide the steering feeling closer to real-world conditions) were added to driver-in-the-loop system to make the driving simulation more realistic (Figure 1).

One phenomenon closely involved with simulator sickness is illusory self-motion due to a visual input, known as 'vection' which is included in the simulated task factors. ${ }^{2}$ If the illusory self-motion is due to an inertial input, it is called 'somatogravic illusion'. Kennedy et al. ${ }^{15}$ stated that visual representations of motion affected the vestibular system. Thus, they concluded that the motion patterns represented in the visual displays of simulators may exert influences on the vestibular system. The research literature from various types of vection study, including those involving exposure to virtual environment systems, has shown that motion sickness is a common side effect of viewing visual scenes of self-motion without physical movement. ${ }^{16-19}$ However, while vection is correlated with visually induced sickness, Lawson et al. ${ }^{20}$ maintained that vection is not a necessary trigger of symptoms. People who experience vection are more likely to experience sickness. ${ }^{17,18}$ The inclusion of a motion base is a simulator-related factor that has been shown to affect simulator sickness. ${ }^{2}$ The platform of a simulator is either a fixed base or a motion base. In a fixed-base simulator, information regarding self-motion is provided merely by the visual system. In contrast, a motion-base simulator provides a subset of the inertial forces that would be present during real movement in the vehicle being simulated. ${ }^{10,21}$ In particular, a motionbase simulator can provide two types of inertial cues: acceleration and tilt. ${ }^{13-15}$ High-fidelity motion-base systems are extremely expensive, but they are used in specific applications (e.g. flight simulators) to enhance the sense of self-motion provided by the visual display. ${ }^{10}$ However, a motion-base simulator can provide motion cues compatible with the initial, but not sustained, acceleration. ${ }^{10}$ For example, forward acceleration can be simulated by pitching the base backwards (tilt coordination) while also translating it forwards (onset acceleration) slightly. ${ }^{10}$

Visual movement through a simulated environment that is not accompanied by the normal inertial cues (e.g. forces and accelerations) associated with movement through the real environment might induce motion sickness, ${ }^{10,11,13}$ particularly nausea. ${ }^{22,23}$ For example, motion sickness has been defined in terms of metrics relating vomiting incidents. ${ }^{22,23}$ Consequently, the overall incidence of simulator sickness is typically lower in simulators with a motion base than in those with a fixed base. ${ }^{12}$ Kennedy et al. ${ }^{14}$ suggested that one of the reasons that simulator sickness incidence was lower in motion-base simulators than in fixed-base simulators was because of the differences in the pilot's head movements during exposure. They explained that, in a moving-base simulator, the pilot's head movements were similar to those in the actual vehicle whereas the head movements in fixed-base simulators were often in conflict with the inertial stimulus, which augmented the contradiction of the simulation. There have been, however, a few reports that contradict the general findings of a difference between the sickness incidence in a fixed-base simulator and that in a motion-base simulators. For example, a study by McCauley and Sharkey ${ }^{13}$ obtained a relatively similar incidence of simulator sickness in a motion-base helicopter simulator to that in a fixed-base simulator.

The proposed approach consists of evaluating the motion sickness at the driver level. It can be explained as vestibular dynamics (longitudinal, lateral and vertical accelerations) measurement from the right-ear level of the subjects via the XSens motion tracker, which is connected to a headphone. The longitudinal, lateral and vertical accelerations of the head movements were measured for this approach and converted into the illness rating by using the motion sickness dose value (MSDV) approach. ${ }^{22}$ In the literature, to be able to rate the simulator sickness, the simulator sickness questionnaire, ${ }^{9}$ the motion sickness questionnaire ${ }^{24}$ and the biofeedback method ${ }^{25}$ are commonly applied with some other approaches such as the MSDV. ${ }^{22,23}$

Although the driving simulator's main utilization domain is training, it can be used to evaluate the motion and simulator sickness. We focused on the represented dynamics fidelity (i.e. how close the dynamics are to the real vehicle dynamics) for two platform conditions and their effects on the participants' vestibular- 
level-sensed illness ratings (via the objective measures shown later in Figure 3), the subjective reports and the correlation of subjective and objective data.

Kennedy et al. ${ }^{26}$ studied the visually induced motion sickness. They reported that the contribution of the elements to simulator sickness is lined up from maximum to minimum in the visually induced motion sickness as $\mathrm{O}>\mathrm{N}>\mathrm{D}$ (where $\mathrm{O}$ represents oculomotor fatigue, $\mathrm{N}$ represents nausea and $\mathrm{D}$ represents disorientation).

Another study, which was conducted by Drexler, ${ }^{27}$ revealed that the contribution order obtained from the simulator sickness questionnaire for driving simulators is $\mathrm{O}>\mathrm{D}>\mathrm{N}$. In our study, the highest principal component was principal component 1 (disorientation-associated sickness, i.e. nausea + dizziness) and the secondhighest principal component was principal component 2 (oculomotor fatigue, i.e. eye strain + tiredness).

Motion sickness was investigated by Gianaros et al. $^{28}$ via a multi-dimensional method by using a motion sickness assessment questionnaire that was presented to participants who were exposed to a rotating optokinetic drum. The results from the motion sickness assessment questionnaire were correlated strongly with the overall scores from the Pensacola diagnostic index $(r=0.81 ; p<0.001)$ and the nausea profile $(r=0.92$; $p<0.001){ }^{28}$ It was found that not only is the motion sickness assessment questionnaire a valid evaluation tool but also it is advantageous to use this multidimensional questionnaire rather than the onedimensional form. ${ }^{28}$

The advantage of the principal component analysis (PCA) method in our study seems to be its capability to simplify the interpretation of the multi-dimensional correlations compared with the factor analysis method. Depending on the PCA method, it was revealed that the subjective self-report analysis was negatively correlated between the static platform case and the dynamic platform case for principal component 1 (the highest principal component which was totally made up of the disorientation-related sickness regarding nausea and dizziness). The study by Gianaros et al. ${ }^{28}$ shows a similarity to our investigation in terms of the sickness profile in which nausea is a principal factor.

During the driving simulator experiments, the same driving scenario of a double-lane-change manoeuvre with a constant velocity of $60 \mathrm{~km} / \mathrm{h}$ with the same conditions (namely the same vehicle model, longitudinal velocity and terrain) was used on a static platform and on a dynamic platform with the software SCANeRstudio version 1.1 from OKTAL.

\section{Motion sickness dose value}

The MSDV is one of the methods used to objectify the motion sickness ratings and was defined in accordance with ISO 2631-1:1997. ${ }^{22}$ In that work, an illness rating method, derived from the MSDV, was utilized. The mathematical expression for the MSDV is given later in equation (5). According to ISO 2631-1:1997, the r.m.s. acceleration values on all axes are defined to reflect more closely the health hazard to which the human body is exposed. The coefficients are described by ISO 2631-1:1997 on the basis of the frequency and the direction of vibration to which the body is exposed. The coefficients $w_{k}=0.426$ (cephalocaudal axis) and $w_{d}=0.067$ (anteroposterior and mediolateral axes) were used to obtain the frequency-weighted r.m.s. acceleration on all the axes (see equation (1)). For evaluation of the health effects, $k_{x}=1.4, k_{y}=$ 1.4 and $k_{z}=1$ (for the longitudinal direction, the lateral direction and the vertical direction respectively) are chosen from the work by Abercromby et al. ${ }^{29}$ The r.m.s. illness rating values at a vestibular (subjects' head movements) level were computed by substituting the $a_{x}, a_{y}$ and $a_{z}$ values for the vestibular level ${ }^{23,29,30}$ according to

$$
\begin{aligned}
a_{R M S} & =\sqrt{\left(k_{x} w_{k} a_{x}\right)^{2}+\left(k_{y} w_{d} a_{y}\right)^{2}+\left(k_{z} w_{d} a_{z}\right)^{2}} \\
a_{x R M S} & =\sqrt{\left(k_{x} w_{k} a_{x}\right)^{2}} \\
a_{y R M S} & =\sqrt{\left(k_{y} w_{d} a_{y}\right)^{2}} \\
a_{z R M S} & =\sqrt{\left(k_{z} w_{d} a_{z}\right)^{2}} \\
\operatorname{MSDV}_{t o t} & =\left[\int_{0}^{t} a_{R M S}^{2}(t) \mathrm{d} t\right]^{0.5} \mathrm{~m} / \mathrm{s}^{3 / 2} \\
I R_{x} & =\frac{1}{50} \operatorname{MSDV}_{x} \\
= & {\left[\int_{0}^{t} a_{x R M S}^{2}(t) \mathrm{d} t\right]^{0.5} \mathrm{~m} / \mathrm{s}^{3 / 2} }
\end{aligned}
$$

where $\operatorname{MSDV}_{\text {tot }}\left(\mathrm{m} / \mathrm{s}^{3 / 2}\right)$ is the total motion sickness dose value ${ }^{23}$ and $a_{R M S}\left(\mathrm{~m} / \mathrm{s}^{2}\right)$ is the r.m.s. acceleration. The longitudinal illness rating is calculated from equation (6). The lateral and the vertical illness ratings are calculated using the same process as in equation (6) by utilizing the corresponding accelerations. The total illness rating, denoted $\mathrm{IR}_{\text {tot }}$, deduced from the MSDV is given by

$$
I R_{t o t}=\frac{1}{50} \mathrm{MSDV}_{t o t}
$$

According to the results obtained for the illness rating $\left(\mathrm{m} / \mathrm{s}^{3 / 2}\right)$, the illness rating scores are ranked as $0,1,2,3$ and greater than 3 as follows. ${ }^{22,23}$

Illness rating $=0$ : I felt good.

Illness rating $=1$ : I felt a mild illness.

Illness rating $=2$ : I felt very bad.

Illness rating $\geqslant 3$ : I felt absolutely terrible. 


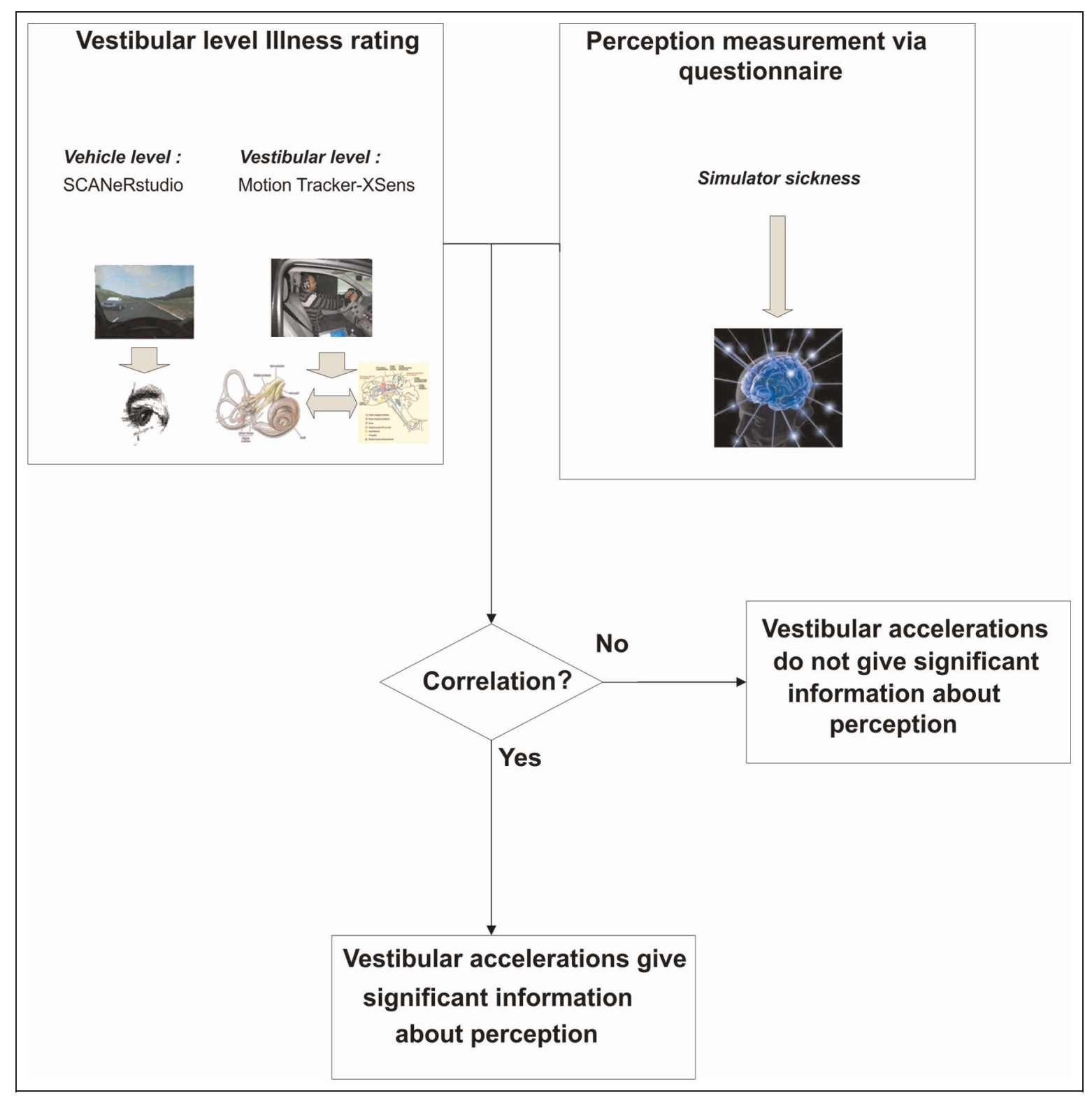

Figure 2. Procedure to correlate the illness rating with the perception of the drivers.

\section{Methods and materials}

\section{Proposed approach}

This study was carried out to understand and rate the effect of platform activation in terms of objective assessments as well as subjective assessments.

Figure 2 shows the approach that we used for enabling the correlation (the Pearson correlation with a confidence interval of $95 \%$ ) between objective data and subjective data in this research to be made. According to this figure, we computed the illness ratings on a vestibular level during the execution of the driving experiments on the SAAM.

The SCANeRstudio and X-Sens measurements are separate measures obtained as from a vehicle level (a vehicle model which moves in the visual environment) and as from a vestibular level (head dynamics from the right-ear alignment) respectively. Vehicle-level dynamics from the visual environment also affect the vestibular-level dynamics. The vestibular-level dynamics of the drivers are influenced only by the vehicle-level dynamics for the static platform case whereas the drivers' vestibular-level dynamics are affected by both the vehicle-level dynamics and the inertial-level dynamics (hexapod motion platform) for the dynamic platform condition.

\section{Experimental set-up}

In this study, the perception of motion sickness was evaluated objectively and subjectively in the SAAM during operating the platform statically and dynamically (see Figure 1 for the multi-sensory integration and motion sickness mechanisms). Table 1 gives the capabilities of the SAAM used for the experiments for the dynamic operations. 
Table I. Limits for each degree of freedom of the SAAM. ${ }^{6,8}$

\begin{tabular}{llll}
\hline Degree of freedom & Displacement & Velocity & Acceleration \\
\hline Pitch & $\pm 22^{\circ}$ & $\pm 30 \mathrm{deg} / \mathrm{s}$ & $\pm 500 \mathrm{deg} / \mathrm{s}^{2}$ \\
Roll & $\pm 21^{\circ}$ & $\pm 30 \mathrm{deg} / \mathrm{s}$ & $\pm 500 \mathrm{deg} / \mathrm{s}^{2}$ \\
Yaw & $\pm 22^{\circ}$ & $\pm 40 \mathrm{deg} / \mathrm{s}$ & $\pm 400 \mathrm{deg} / \mathrm{s}^{2}$ \\
Heave & $\pm 0.18 \mathrm{~m}$ & $\pm 0.30 \mathrm{~m} / \mathrm{s}$ & $\pm 0.5 \mathrm{~g}$ \\
Surge & $\pm 0.25 \mathrm{~m}$ & $\pm 0.5 \mathrm{~m} / \mathrm{s}$ & $\pm 0.6 \mathrm{~g}$ \\
Sway & $\pm 0.25 \mathrm{~m}$ & $\pm 0.5 \mathrm{~m} / \mathrm{s}$ & $\pm 0.6 \mathrm{~g}$ \\
\hline
\end{tabular}

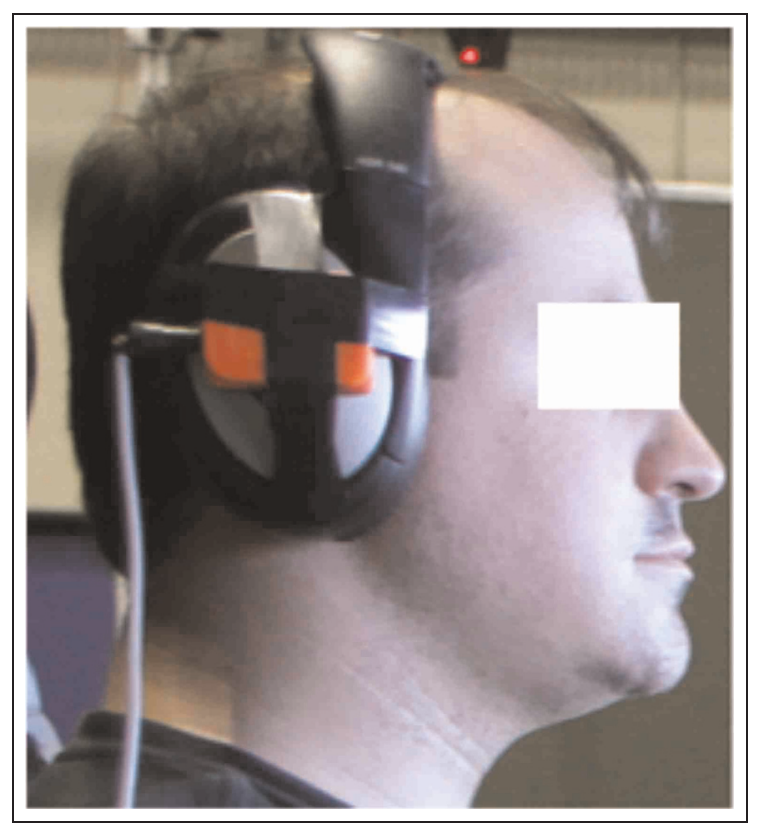

Figure 3. Vestibular-level data acquisition.

The SAAM simulator was designed and developed by Arts et Metiers Paris Tech and Renault. The objective rating refers to the methods performed through measurements of those parameters which do not contain personal assessments. In the case of the subjective assessment of simulator sickness, we proposed a method called 'perception due to psychophysics'.

For the objective evaluation, the perceived dynamics by the subjects during the real-time sessions were observed regarding the vestibular level. The advantage of the data acquisition module from the SCANeRstudio version 1.1 was that the data related to commands (steering-wheel angle, accelerator, brake pedal force, etc.), dynamics (vehicle dynamics data), engine and frequency analysis (data collection in real time via SCANeRstudio) can be saved, whereas the record of the sensed dynamics on the vestibular level of the driver during the online tests was obtained by using a threedimensional acceleration sensor (XSens) attached to a headphone aligned to the driver's right ear (Figure 3).

\section{Protocol}

Figure 4 illustrates the bird's-eye view of the trajectory which was driven during the driving simulator

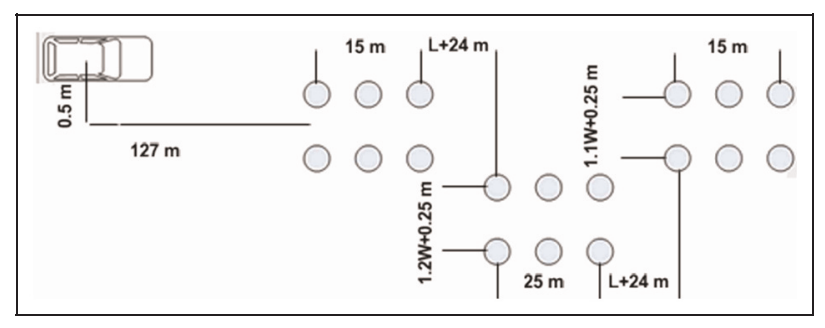

Figure 4. NATO chicane right turn used on the driving simulator session. ${ }^{31}$

experiment. In Figure 4, the starting location of the vehicle tested on the driving simulator experiments as well as the distances between the pylons are depicted. $W$ and $L$ are the width and the length respectively of the vehicle. Here $W=1 \mathrm{~m}$ and $L=1.5 \mathrm{~m}$.

The subjects were asked to drive a NATO chicane manoeuvre (see Figure 4). ${ }^{31,32}$ Different conditions were evaluated with or without the motion platform. After each attempt, the subjects were asked to fill in the available questionnaire for the subjective rating of the perception due to psychophysics. Also, during each trial, the data were recorded with a sampling period of $0.05 \mathrm{~s}$. The subjects drove the same scenario twice to become familiarized with it before the evaluation phase. The tests were accomplished in different conditions with the NATO chicane scenario at a constant driving velocity $V_{x}=60 \mathrm{~km} / \mathrm{h}$ for a duration of $37-50 \mathrm{~s}$.

Table 2 shows the parameters of the motion cueing algorithm used in the dynamic platform simulator condition within these experiments.

The second-order low-pass (LP) cut-off frequency and the second-order LP damping factor are the onset cues for the tilting (pitch and roll) as the sustained part of the movements. The first-order LP time constant illustrates the time delays for the sustained accelerations of the motion drive algorithm. The sustained part of the motion is associated with the cue conflicts between the visual (cabin- or vehicle-level) environment and the inertial (motion-platform-level) environment for lowfrequency motion (tilting, i.e. pitch and roll).

The second-order high-pass (HP) cut-off frequency and the second-order HP damping factor are the onset cues for the yaw motion and the longitudinal and lateral translational accelerations. The first-order HP time constant gives the time delays for the onset cues. The onset cues of the motion are related to the cue conflicts between the visual (cabin- or vehicle-level) environment and the inertial (motion-platform-level) environment for highfrequency motion (translational accelerations and yaw).

\section{Subjects}

20 subjects (18 males and two females) with an age distribution of $28.6 \pm 5.97$ years old and a driving licence experience distribution of $9.4 \pm 6.11$ years participated in the experiments. The limitation of the current investigation is that no gender effect was taken into account. 
Table 2. Classical motion cueing algorithm parameters. ${ }^{6,8}$

\begin{tabular}{|c|c|c|c|c|c|}
\hline Parameter (units) & Longitudinal & Lateral & Roll & Pitch & Yaw \\
\hline Second-order LP cut-off frequency $(\mathrm{Hz})$ & & & 0.3 & 0.7 & \\
\hline Second-order LP damping factor & & & 0.3 & 0.7 & \\
\hline First-order LP time constant (s) & 0.1 & 0.1 & & & 0.1 \\
\hline Second-order HP cut-off frequency $(\mathrm{Hz})$ & 0.5 & 0.5 & & & 2 \\
\hline Second-order HP damping factor & I & 1 & & & l \\
\hline First-order HP time constant (s) & 2 & 2 & & & 2 \\
\hline
\end{tabular}

LP: low-pass; HP: high-pass.

The subjects were researchers, engineers and students from the laboratory. The questionnaires were administered to the subjects in the French language. They had a familiarization drive on the driving simulator before each session to check for any predisposition regarding motion sickness, to avoid misevaluation and to help them to assess the procedure as objectively as possible.

\section{Objective measures}

Objective evaluation refers to an assessment method for driving simulator applications in which various data are measured. In this investigation, according to the proposed approach, the measurements were realized for vestibular cues (measurements of the head movements of the subjects) to obtain the illness rating which is derived from the MSDV for changing the motion base activation of the SAAM. Vestibular-level data acquisition (head movements of the participants) is performed via a sensor which measures the roll, the pitch, the yaw angles and the accelerations along the $x, y$ and $z$ axes. The data are calibrated with respect to the 'threedimensional quaternion orientation'. The sampling period for the data registration during the sensor measurements is $0.05 \mathrm{~s}$. For the calibrated data acquisition, the alignment reset was chosen, which simply combines the object reset and the heading reset at a single instant in time. This has the advantage that all coordinate systems can be aligned with a single action. In this study, we utilized the longitudinal, lateral and vertical accelerations from the head (vestibular) levels of the participants (see Figure 3). The r.m.s. accelerations were computed from equations (2), (3) and (4). Then, the component illness rating metrics were obtained for the longitudinal direction $x$, the lateral direction $y$ and the vertical direction $z$. For example, equation (6) gives the illness rating of the longitudinal accelerations measured by the subjects' heads (vestibular, Figure 3) levels as a component illness rating for longitudinal dynamics. We expanded the component illness rating analysis for the lateral and vertical accelerations by substituting the related r.m.s. accelerations.

We defined the compound illness rating as $\mathrm{IR}_{t o t}$, and the compound r.m.s. acceleration was obtained by substituting the measured vestibular level longitudinal $(x)$, lateral $(y)$ and vertical $(z)$ accelerations in equation (1).
In order to determine the compound illness rating $\mathrm{IR}_{\text {tot }}$ which is given in equation (7), equation (1) was substituted in equation (5), and finally equation (5) was replaced in equation (7).

\section{Subjective measures}

The questions regarding the perception due to psychophysics that were asked just after the completion of each trial are the following. ${ }^{8}$

In order to assess the driving simulator tests subjectively on behalf of the perception due to psychophysics, a simulator sickness and psychophysical perception questionnaire which consists of 12 questions (graded from 1: very little $\rightarrow 10$ : very strong) was given to each participant to obtain a subjective measure of the driving simulation. We named it perception due to psychophysics, which is rather different from the existing simulator sickness questionnaire ${ }^{9}$ and the motion sickness history questionnaire ${ }^{24}$, not only because of the disorientation-related sickness criteria (nausea and dizziness) but also because we aimed to assess the subjective impressions of the participants in terms of perceiving the stimuli and their reactions to them (psychophysics) such as fear, mental pressure, fatigue and anxiety (Table 3).

In the simulator sickness questionnaire, questions about nausea, disorientation and oculomotor fatigue were used, and their evaluation rating scales were divided into four. ${ }^{9}$ In our questionnaire set, we used a grading scale between 1 and 10 .

\section{Data analysis}

The PCA method is a statistical technique used for dimensionality reduction and representation of multivariate data sets represented by an $N \times p$ matrix $\mathbf{X}$ with $N$ observations and $p$ variables. ${ }^{33}$ This method transforms a multi-variate data set of intercorrelated variables into a data set of new uncorrelated linear combinations of the original variables. Before the analysis, the columns of $\mathbf{X}$ are centred if variables have very heterogeneous variances. In addition to centring, when the variables are measured with different units, it is customary to standardize each variable to the unit norm. This is obtained by dividing each variable by its norm. 
Table 3. Questionnaire on the perception due to psychophysics.

\begin{tabular}{|c|c|}
\hline Question number & Question (question rating) \\
\hline Q। & $\begin{array}{l}\text { Were you prone to vomit? } \\
\text { (I: very little } \rightarrow 10 \text { : very strong) }\end{array}$ \\
\hline Q2 & $\begin{array}{l}\text { Did you feel nauseous? } \\
\text { (I: very little } \rightarrow \text { I0: very strong) }\end{array}$ \\
\hline Q3 & $\begin{array}{l}\text { Did you have a cold sweat? } \\
\text { (I: very little } \rightarrow 10 \text { : very strong) }\end{array}$ \\
\hline Q4 & $\begin{array}{l}\text { Did you feel dizzy? } \\
\text { (I: very little } \rightarrow \text { I0: very strong) }\end{array}$ \\
\hline Q5 & $\begin{array}{l}\text { Did you feel eye strain? } \\
\text { (I: very little } \rightarrow \text { 10: very strong) }\end{array}$ \\
\hline Q6 & $\begin{array}{l}\text { Did you have trouble with your eyes? } \\
\text { (I: very little } \rightarrow 10 \text { : very strong) }\end{array}$ \\
\hline Q7 & $\begin{array}{l}\text { Did you have a headache? } \\
\text { (I: very little } \rightarrow \mid 0 \text { : very strong) }\end{array}$ \\
\hline Q8 & $\begin{array}{l}\text { Did you feel mental pressure? } \\
\text { (I: very little } \rightarrow \text { I0: very strong) }\end{array}$ \\
\hline Q9 & $\begin{array}{l}\text { Did you feel fear? } \\
\text { (I: very little } \rightarrow 10 \text { : very strong) }\end{array}$ \\
\hline Q10 & $\begin{array}{l}\text { Were you bored? } \\
\text { (I: very little } \rightarrow 10 \text { : very strong) }\end{array}$ \\
\hline QII & $\begin{array}{l}\text { Were you tired? } \\
\text { (I: very little } \rightarrow \text { I0: very strong) }\end{array}$ \\
\hline Q12 & $\begin{array}{l}\text { Did you feel anxiety (uneasiness)? } \\
\text { (I: very little } \rightarrow 10 \text { : very strong) }\end{array}$ \\
\hline
\end{tabular}

In this case, the analysis is referred to as a correlation PCA.

For a given $p$-dimensional data set, the PCA method applies an orthogonal transformation on an $m$-dimensional subspace $(1<m<p)$ spanned by orthogonal axes called principal axes along which the variance of the data is maximized. The principal axes can be given by the leading eigenvectors associated with the $m$ largest eigenvalues of the correlation matrix of the data set. The values of the data along these axes are called principal components. In fact, a small number of principal components can describe most of the variance in the original data set and are therefore used as features to replace the original data representation. The use of these principal components as features can reduce the dimensions of the data representation without much loss of the variance in the original data set.

As the variables are standardized to the unit norm, they can be represented by vectors lying on an $m$ dimensional unit sphere, which we can project on the first two principal axes. These projections are vectors whose coordinates are correlations between variables with the first two principal components.

Therefore the variables will be positioned inside a circle called 'the circle of correlations', which is useful to evaluate the affinities and antagonisms between variables. The closer a variable is to the circle of correlations, the better we can reconstruct this variable from the first two components. In this case, if the variables are close to each other, they are significantly positively correlated ( $r$ close to 1 ); if they are orthogonal, they are not correlated ( $r$ close to 0$)$; if they are on the opposite side of the centre, then they are significantly negatively correlated ( $r$ close to -1 ). When a variable is closer to the centre of the circle, this means that it is less important for the first two components.

In this study, the perception of motion sickness (psychophysical subjective assessments) was compared by using the PCA method for the static simulator condition and the dynamic simulator condition. The questionnaires only about psychophysical perception for the two conditions (the static case and the dynamic platform case) were evaluated by using a two-tailed MannWhitney $U$ test and PCA. The inputs to the PCA methods were the perceptual questions given in Table 3 .

The same pattern or symptomatology factor structures were used in both conditions of the simulator platform operations during the experiments.

Afterwards, perceptual convergence to the physical situation was checked by using the Pearson correlation between the objective data and the subjective measure data. In other words, the Pearson correlation method was employed to evaluate the effects of the objective measures $\left(\mathrm{IR}_{x}, \mathrm{IR}_{y}, \mathrm{IR}_{z}\right.$ and $\mathrm{IR}_{\text {tot }}$ values computed from the vestibular-level longitudinal, lateral and vertical acceleration measurements given in Figure 3) on the self-reports (the subjective measures via the questionnaire in Table 3 ).

\section{Results and discussion}

Table 4 illustrates the self-report results of using two conditions of a driving simulator, namely with a static platform or with a dynamic platform. Statistically significant mean differences exist between the treatments for nausea, dizziness, eye strain and fatigue (tiredness) in the static condition and the dynamic condition.

The two-tailed Mann-Whitney $U$ test was chosen to compare the significance in the differences between the individual questions rather than the PCA.

As seen from the two-tailed Mann-Whitney $U$ test, experiencing nausea $(p=0.019<0.05)$ and dizziness $(p=0.018<0.05)$ were decreased significantly from the static case to the dynamic case.

Also, as found from the two-tailed Mann-Whitney $U$ test, eye strain $(p=0.047<0.05)$ and tiredness $(p=$ $0.047<0.05)$ were reduced significantly from the static case to the dynamic case.

In our context, the statistical analysis was accomplished by using the language $\mathrm{R}$ for both objective assessments and subjective assessments. On the righthand side of Figure 5, we see that the first two eigenvalues (black bars) of the correlation matrix correspond to $63.42 \%$ and $13.38 \%$ respectively of the variance (see Table 5). It appears that most of the information carried by the variables can be extracted from only the first two principal axes owing to the PCA carried out by $\mathrm{R}$ statistical analysis software. On the left-hand side of Figure 5, the subjective evaluation data were projected on the correlation circle on the first two principal axes 
Table 4. Self-report results.

\begin{tabular}{|c|c|c|c|}
\hline \multirow[t]{2}{*}{ Self-report (question number) } & \multicolumn{2}{|c|}{ Value for the self-report for the following platforms } & \multirow{2}{*}{$\begin{array}{l}p \text { value from a two-tailed } \\
\text { Mann-Whitney } U \text { test }\end{array}$} \\
\hline & $\begin{array}{l}\text { Static platform } \\
\text { (mean } \pm \text { standard deviation) }\end{array}$ & $\begin{array}{l}\text { Dynamic platform } \\
\text { (mean } \pm \text { standard deviation) }\end{array}$ & \\
\hline Propensity to vomit (QI) & $2.500 \pm 1.225$ & $1.429 \pm 0.787$ & $p=0.067>0.05$ \\
\hline Nausea (Q2) & $3.000 \pm 1.265$ & $1.57 \mid \pm 0.535$ & $p=0.019<0.05$ \\
\hline Cold sweat (Q3) & $2.000 \pm 0.632$ & $1.429 \pm 0.787$ & $p=0.120>0.05$ \\
\hline Dizziness (Q4) & $2.667 \pm 1.211$ & $1.429 \pm 0.535$ & $p=0.018<0.05$ \\
\hline Eye strain (Q5) & $2.167 \pm 0.983$ & $1.286 \pm 0.488$ & $p=0.047<0.05$ \\
\hline Trouble with eyes (Q6) & $2.000 \pm 0.894$ & $1.429 \pm 0.535$ & $p=0.214>0.05$ \\
\hline Headache (Q7) & $2.000 \pm 0.894$ & $1.286 \pm 0.488$ & $p=0.114>0.05$ \\
\hline Mental pressure (Q8) & $1.667 \pm 0.816$ & $1.429 \pm 0.535$ & $p=0.630>0.05$ \\
\hline Fear (Q9) & $1.500 \pm 0.548$ & $1.429 \pm 0.535$ & $p=0.805>0.05$ \\
\hline Boredom (QI0) & $2.333 \pm 1.862$ & $1.286 \pm 0.488$ & $p=0.148>0.05$ \\
\hline Tiredness (QII) & $2.000 \pm 0.632$ & $1.286 \pm 0.488$ & $p=0.047<0.05$ \\
\hline Anxiety (uneasiness) (Q $\mid 2$ ) & $2.167 \pm 0.753$ & $1.429 \pm 0.535$ & $p=0.074>0.05$ \\
\hline
\end{tabular}

Table 5. Inertias of the eigenvalues and the percentages in the variance.

\begin{tabular}{lll}
\hline $\begin{array}{l}\text { Prinicipal } \\
\text { component }\end{array}$ & $\begin{array}{l}\text { Inertia of the } \\
\text { eigenvalues }\end{array}$ & $\begin{array}{l}\text { Percentage in } \\
\text { the variance(\%) }\end{array}$ \\
\hline Component I & 15.22 & 63.42 \\
Component 2 & 3.21 & 13.38 \\
Component 3 & 2.54 & 10.58 \\
Component 4 & 1.29 & 5.38 \\
Component 5 & 0.9 & 3.75 \\
Component 6 & 0.48 & 2 \\
Component 7 & 0.23 & 0.96 \\
Component 8 & 0.096 & 0.53 \\
\hline
\end{tabular}

associated with principal component 1 (horizontal axis) (labelled Comp1 in Figure 5) and principal component 2 (vertical axis) (labelled Comp2 in Figure 5) respectively. Most of the original projected variables had a good representation on the principal plane generated by the first two principal axes. Moreover, according to the principal axis associated with principal component 1 , it was demonstrated that the subjective data were positively correlated with principal component 1 for the dynamic platform, whereas these data were negatively correlated with principal component 1 in the case of the static platform. This means that the simulator sickness perception revealed from the questionnaire on the perception due to psychophysics (Table 3) showed conflict between the static platform and the dynamic platform. Principal component 1 refers to the disorientationrelated sickness (nausea and dizziness; see Table 3), whereas principal component 2 is dedicated to oculomotor fatigue and illness (eye strain and tired; see Table 3).

Objective data were computed from the illness ratings (equation (7)) on the vestibular level during the execution of the driving experiments on the SAAM, and subjective data were extracted from responses to a questionnaire on both the static platform condition and the dynamic platform condition. The approach that we used to prove the correlation between objective data and subjective data in this research is the Pearson correlation.

The subscripts $S T A$ and $D Y N$ signify the static condition and the dynamic condition respectively for the answers given by the subjects to the perception questionnaire in Tables 6 and 7.

So as to measure the driver's perception, a perception due to psychophysics questionnaire was introduced to obtain an opinion on the subjective evaluation of simulator sickness. Reason and Brand ${ }^{34}$ suggested that a significant reduction in motion sickness occurs when an individual adopts a postural position. The postural position refers to the spinal stability when the participants are standing up just after the experimental sessions or at the change between pre- and postexperimental sessions. Riccio and Stoffregen ${ }^{35}$ introduced the 'postural instability theory' also to define the relations between perception and the control of action (head movements in our study). This approach considers the behaviour of the individual as fundamental in motion sickness aetiology. The postural instability theory of motion sickness presumes that motion sickness results from and can be estimated by instabilities in controlling the spine. This was attributed to constraints in motion of the head. Kennedy et al. ${ }^{14}$ and Reason and Brand $^{34}$ described the relations between the head motions and motion sickness through the Coriolis mechanisms (with actual inertial cues, i.e. the motion platform) and pseudo-Coriolis (through visual cues, i.e. the static operation of the simulator) stimulation. Coriolis stimulation occurs when the head is tilted out of the axis of rotation during actual body rotation. ${ }^{36-40}$ Pseudo-Coriolis stimulation occurs when the head is tilted as perceived self-rotation that is induced by visual stimuli. $^{38}$

According to Kennedy et al., ${ }^{14}$ the subjects' head movements in a moving-base simulator were similar to those in the actual vehicle, ${ }^{36-40}$ whereas the head movements in fixed-base simulators were often in conflict 


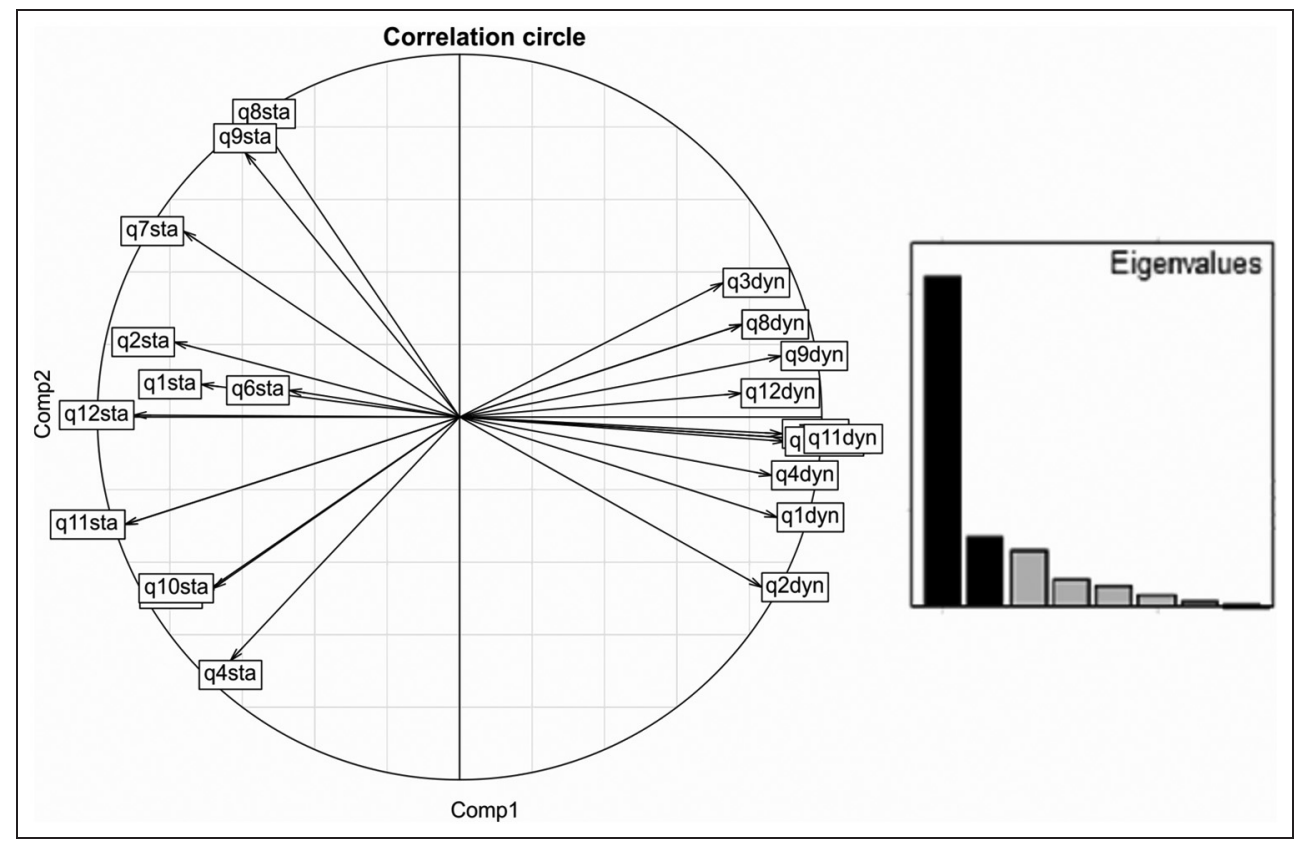

Figure 5. Correlation circle and eigenvalues of the PCA for the static condition and the dynamic condition. Compl: principal component I (horizontal axis); Comp2: principal component 2 (vertical axis).

Table 6. Correlation coefficients between the objective data and the subjective data for the static platform.

\begin{tabular}{|c|c|c|c|c|}
\hline Variable & $\mathrm{IR}_{x}$ & $\mathrm{IR}_{y}$ & $\mathbb{I R}_{\mathrm{z}}$ & $\mathbb{I R}_{\text {tot }}$ \\
\hline$q_{\text {ISTA }}$ & 0.625 & 0.108 & -0.302 & 0.538 \\
\hline$q_{2 S T A}$ & 0.453 & -0.145 & -0.211 & 0.385 \\
\hline$q_{3 \text { STA }}$ & 0.836 & 0.213 & 0.367 & 0.855 \\
\hline$q_{4 S T A}$ & 0.922 & 0.006 & 0.602 & 0.970 \\
\hline$q_{5 S T A}$ & 0.948 & 0.175 & 0.363 & 0.958 \\
\hline$q_{6 S T A}$ & 0.661 & 0.054 & -0.275 & 0.575 \\
\hline$q_{7 \text { STA }}$ & 0.135 & -0.012 & -0.561 & 0.029 \\
\hline$q_{8 S T A}$ & -0.308 & 0.174 & -0.617 & -0.391 \\
\hline$q_{9 S T A}$ & -0.335 & 0.300 & -0.681 & -0.424 \\
\hline qIOSTA & 0.984 & 0.187 & 0.134 & 0.953 \\
\hline qIISTA & 0.836 & 0.213 & 0.367 & 0.855 \\
\hline$q_{12 S T A}$ & 0.613 & 0.149 & 0.134 & 0.603 \\
\hline
\end{tabular}

The values in bold are different from 0 with a significance level $\alpha=0.05$.

with the inertial stimulus, which increased the discrepancy of the simulation. ${ }^{38}$ Those findings are parallel to ours (Table 6 and Table 7).

This work enabled us to answer some of the research questions asked.

Q3 (having a cold sweat) was significantly correlated with the $\mathrm{IR}_{y}$ for the dynamic platform condition $(p<0.05)$, whereas it was significantly correlated with $\operatorname{IR}_{x}$ and $\operatorname{IR}_{t o t}$ in the static condition $(p<0.05)$. Q4 (feeling dizzy) was significantly correlated with $\mathrm{IR}_{z}$ for the dynamic platform, whereas it was significantly correlated with $\mathrm{IR}_{x}$ and $\mathrm{IR}_{t o t}$ for the static case $(p<0.05)$. Q5 (feeling eye strain) was significantly correlated with $\mathrm{IR}_{z}$ and $\mathrm{IR}_{\text {tot }}$ at the dynamic platform, while it was significantly correlated with $\mathrm{IR}_{x}$ and $\mathrm{IR}_{\text {tot }}$ at the static platform $(p<0.05)$. Q7
Table 7. Correlation coefficients between the objective data and the subjective data for the dynamic platform.

\begin{tabular}{|c|c|c|c|c|}
\hline Variable & $\mathbb{I R}_{x}$ & $\mathbb{I R}_{y}$ & $\mathrm{IR}_{\mathrm{z}}$ & $\mathrm{IR}_{\text {tot }}$ \\
\hline$q_{\text {IDYN }}$ & -0.291 & -0.262 & 0.112 & -0.292 \\
\hline$q_{2 D Y N}$ & 0.496 & -0.250 & 0.694 & 0.498 \\
\hline$q_{3 \mathrm{DYN}}$ & -0.283 & 0.902 & -0.114 & -0.221 \\
\hline$q_{4 D Y N}$ & 0.409 & -0.259 & 0.765 & 0.416 \\
\hline$q_{5 D Y N}$ & 0.730 & 0.118 & 0.906 & 0.758 \\
\hline$q_{6 D Y N}$ & 0.415 & 0.597 & 0.599 & 0.468 \\
\hline$q_{7 D Y N}$ & 0.730 & 0.118 & 0.906 & 0.758 \\
\hline$q_{8 D Y N}$ & 0.415 & 0.597 & 0.599 & 0.468 \\
\hline$q_{9 D Y N}$ & 0.415 & 0.597 & 0.599 & 0.468 \\
\hline$q_{\text {IODYN }}$ & 0.730 & 0.118 & 0.906 & 0.758 \\
\hline$q_{\text {IIDYN }}$ & 0.730 & 0.118 & 0.906 & 0.758 \\
\hline$q_{12 \mathrm{DYN}}$ & 0.415 & 0.597 & 0.599 & 0.468 \\
\hline
\end{tabular}

The values in bold are different from 0 with a significance level $\alpha=0.05$.

(having a headache) was significantly correlated with $\mathrm{IR}_{z}$ and $\mathrm{IR}_{\text {tot }}$ for the dynamic situation $(p<0.05)$. The other component and compound illness ratings had no significant correlation with Q7 $(p>0.05)$ for the static case. Q10 (feeling bored) was significantly correlated with the $\mathrm{IR}_{z}$ and $\mathrm{IR}_{\text {tot }}$ for the dynamic situation, while it was significantly correlated with $\mathrm{IR}_{x}$ and $\mathrm{IR}_{\text {tot }}$ for the static case $(p<0.05)$. Q11 (feeling tired) was significantly correlated with the $\mathrm{IR}_{x}$ and $\mathrm{IR}_{\text {tot }}$ for the static situation and it was significantly correlated with $\mathrm{IR}_{z}$ and $\mathrm{IR}_{\text {tot }}$ for the dynamic case $(p<0.05)$.

The two-tailed Mann-Whitney $U$ test indicated that nausea $(\mathrm{Q} 2: U(20), p=0.019<0.05)$ and dizziness (Q4: $U(20), p=0.018<0.05$ ) abated significantly from the static condition to the dynamic condition. 
Similarly the two-tailed Mann-Whitney $U$ test suggests that eye strain (Q5: $U(20), p=0.047<0.05)$ and fatigue (Q11: $U(20), p=0.047<0.05)$ were reduced significantly by using the dynamic platform rather than the static platform.

\section{Conclusion}

The subjective evaluation comparison showed that the answers to the questionnaires for the static case were negatively correlated with the answers given to the same questionnaires for the dynamic platform for the highest principal component (PCA, principal component 1, i.e. nausea + dizziness) (the percentage in the variance is $63.42 \%$ ). This already indicates a difference for the contribution of the dynamic platform. In order to evaluate the perception fidelity, the Pearson correlation between the subjective data and the objective data was checked.

Having a cold sweat (fear indicator), feeling dizzy (disorientation), feeling eye strain (visual sickness), feeling bored (psychophysical situation) and feeling tired (psychophysical situation) were significantly positive correlated with $\mathrm{IR}_{x}$ and $\mathrm{IR}_{\text {tot }}$ for the static platform. These results for the static case showed that the main factor which induced the discomfort was the longitudinal head (vestibular-level) dynamics. While the lateral and the vertical head dynamics were not significant in the perception of the motion sickness, the total compound head dynamics were also significantly correlated with the fear indicator, the disorientation feeling, the visual sickness and the psychophysical situation.

Having a cold sweat was significantly correlated with $\mathrm{IR}_{y}$ for the dynamic case. This showed that the self-report findings are suggestive. Moreover, feeling dizzy, feeling eye strain, feeling bored and feeling tired were positively correlated with $\mathrm{IR}_{z}$ and $\mathrm{IR}_{\text {tot }}$. These findings for the dynamic condition depicted that the main factor which provoked the disorientation, the visual sickness and the psychophysical situation was vertical head dynamics of the subjects. Because the longitudinal and the lateral head dynamics did not have significant roles in the perception of the motion sickness, the total compound vestibular level dynamics were also correlated with the disorientation, the visual sickness and the psychophysical situation.

\section{Acknowledgements}

Arts et Metiers Paris Tech built the SAAM driving simulator in collaboration with Renault and Grand Chalon. This research study was realized in the framework of the geDriver project.

\section{Funding}

This research received no specific grant from any funding agency in the public, commercial or not-for-profit sectors.

\section{Declaration of conflict of interest}

The authors declare that there is no conflict of interest.

\section{References}

1. Kemeny A and Panerai F. Evaluating perception in driving simulation experiments. Trends Cognitive Sci 2003; 7(1): 31-37.

2. Kolasinski EM. Simulator sickness in virtual environments. Report ARI-TR-1027, Army Research Institute for the Behavioural and Social Sciences, Alexandria, Virginia, USA, 1995.

3. Berger DR, Schulte-Pelkum J and Bülthoff HH. Simulating believable forward accelerations on a Stewart motion platform. ACM Trans Appl Perception 2010; 7(1): 5:15:27.

4. Curry R, Artz B, Cathey L, et al. Kennedy SSQ results: fixed-vs motion-based Ford simulators. In: Driving simulation conference, Paris, France, 11-13 September 2002, pp. 289-300.

5. Watson G. A synthesis of simulator sickness studies conducted in a high-fidelity driving simulator. In: Driving simulation conference, Paris, France, 6-8 September 2000, pp. 69-78.

6. Aykent B, Merienne F, Paillot D and Kemeny A. Influence of inertial stimulus on visuo-vestibular cues conflict for lateral dynamics at driving simulators. $J$ Ergonomics 2013; 3: 1-7.

7. Siegler I, Reymond G, Kemeny A, et al. Sensorimotor integration in a driving simulator: contributions of motion cueing in elementary driving tasks. In: Driving simulation conference, Nice, France, 5-7 September 2001, pp. 21-32.

8. Aykent B, Paillot D, Merienne F, et al. Study of the influence of different washout algorithms on simulator sickness for a driving simulation task. In: ASME world conference on innovative virtual reality, Milan, Italy, 2729 June 2011, paper WINVR2011-5545, pp. 331-341. New York: ASME.

9. Kennedy RS, Lane NE, Berbaum KS, et al. Simulator sickness questionnaire: an enhanced method for quantifying simulator sickness. Int J Aviat Psychol 1993: 3(3): 203-220.

10. Durlach NI and Mavor AS. Virtual reality:scientific and technological challenges. Washington, DC: National Academies Press, 1995.

11. May $\mathbf{J}$ and Badcock D. Vision and virtual environments. In: Stanney KM (ed) Handbook of virtual environments: design, implementation, and applications. Mahwah, New Jersey: Lawrence Erlbaum Associates, 2002, pp. 29-64.

12. McCauley M (ed). Research issues in simulator sickness, Proceedings of a workshop. Washington, DC: National Academies Press, 1984.

13. McCauley M and Sharkey T. Cybersickness-perception of self-motion in virtual environments. Presence: Teleoperators Virtual Environ 1992; 1(3): 311-318.

14. Kennedy RS, Berbaum KS, Lilienthal MG, et al. Guidelines for alleviation of simulator sickness symptomatology. Report NAVTRASYSCEN-TR-87-007, Naval Training Systems Center Orlando, Florida, USA, 1987.

15. Kennedy R, Berbaum K, Allgood G, et al. Etiological significance of equipment features and pilot history in simulator sickness. In: Motion cues in flight simulation 
and simulator induced sickness, AGARD Conference Proceedings 443, Brussels, Belgium, 29 September -1 October 1988, 22 pp. Neuilly sur Seine: AGARD.

16. Hettinger LJ. Illusory self-motion in virtual environments. In: Stanney KM (ed) Handbook of virtual environments: design, implementation, and applications. Mahwah, New Jersey: Lawrence Erlbaum Associates, 2002, pp. 471-492.

17. Hettinger LJ, Berbaum KS, Kennedy RS, et al. Vection and simulator sickness. Milit Psychol 1990; 2(3): 171-181.

18. Hettinger LJ and Ricco G. Virtually induced motion sickness in virtual environments. Presence: Teleoperators Virtual Environ 1992; 1(3): 306-310.

19. Yardley L. Motion sickness and perception: a reappraisal of the sensory conflict approach. Br J Psychol 1992; 83(4): 449-471.

20. Lawson B, Graeber D, Mead A, et al. Signs and symptoms of human syndromes associated with synthetic experiences. In: Stanney KM (ed) Handbook of virtual environments: design, implementation, and applications. Mahwah, New Jersey: Lawrence Erlbaum Associates, 2002, pp .589-618.

21. Dizio P and Lackner J. Spatial orientation, adaptation, and motion sickness in real and virtual environments. Presence 1992; 1(3): 319-328.

22. Griffin MJ. Handbook of human vibration. Amsterdam: Elsevier, 1996.

23. ISO 2631-1: 1997. Mechanical vibration and shock: evaluation of human exposure to whole-body vibration. Part 1 , General requirements. Geneva: International Organization for Standardization, 1997.

24. Kennedy RS, Fowlkes JE, Berbaum KS, et al. Use of a motion sickness history questionnaire for prediction of simulator sickness. Aviat, Space, Environ Med 1992; 63(7): 588-593.

25. Kim MS, Moon YG, Kim GD, et al. Partial range scaling method based washout algorithm for a vehicle driving simulator and its evaluation. Int J Automot Technol 2010; 11(2): 269-275.

26. Kennedy RS, Drexler J and Kennedy RC. Research in visually induced motion sickness. Appl Ergonomics 2010; 41: 494-503.

27. Drexler JM. Identification of system design features that affect sickness in virtual environments. Doctoral Dissertation, University of Central Florida, Orlando, Forida, USA, 2006
28. Gianaros PJ, Muth ER, Mordkoff T, et al. A questionnaire for the assessment of the multiple dimensions of motion sickness. Aviat Space Environ Med 2001; 72(2) 115-119.

29. Abercromby A., Amonette W, Layne C, et al. Vibration exposure and biodynamic responses during whole-body vibration training. Med Sci Sports Exercise 2007; 39(10): $1794-1800$

30. Griffin MJ. Minimum health and safety requirements for workers exposed to hand-transmitted vibration and whole-body vibration in the European Union; a review. Occup Environ Med 2004; 61(5): 387-397.

31. Gunter DD, Bylsma WW, Edgar K, et al. Using modeling and simulation to evaluate stability and traction performance of a track-laying robotic vehicle. In: Trevisani DA and Sisti F (eds) Enabling technologies for simulation science IX, Proceedings of the SPIE, Vol 5805, pp. 66-73. Bellingham, Washington: SPIE, 2005.

32. Yang X and Gander J. Driver's preview strategy and its impact on NATO double lane change maneuver. SAE paper 2011-01-980, 2011

33. Husson F, Lê $\mathrm{S}$ and Pagès J. Analyse de données avec $R$. Rennes: Presses Universitaires de Rennes, 2009.

34. Reason J and Brand J. Motion sickness. New York: Academic Press, 1975.

35. Riccio G and Stoffregen T. An ecological theory of motion sickness and postural instability. Ecol Psychol 1991; 3(3): 195-240.

36. Dichgans $\mathbf{J}$ and Brandt $\mathrm{T}$. Optokinetic motion sickness and pseudo-Coriolis effects induced by moving visual stimuli. Acta Oto-Laryngol 1973; 76(1-6): 339-348.

37. Dizio $\mathrm{P}$ and Lackner J. The effects of gravitoinertial force level and head movements on post-rotational nystagmus and illusory after-rotation. Expl Brain Res 1988; 70(3): 485-495.

38. Dizio P and Lackner J. Perceived self-motion elicited by postrotary head tilts in a varying gravitoinertial force background. Attention, Perception, Psychophys 1989; 46(2): 114-118.

39. Guedry FE. Visual control of habituation to complex vestibular stimulation in man. Acta Oto-Laryngol 1964; 58(1-6): 377-389.

40. Guedry FE and Montague EK. Quantitative evaluation of the vestibular Coriolis reaction. Aerospace Med 1961; 32(6): 487-500. 